\title{
Participación CiUdadana en la \\ ARQUITECTURA Y EL URBANISMO. \\ El CASO del BARRIO de LA ISLETA
}

\author{
Citizen Participation in Architecture and Urbanism.
}

The Case of the District of La Isleta

\section{Vicente Javier Díaz García vicente.diaz@ulpgc.es Universidad de Las Palmas de Gran Canaria}

RESUMEN: Para comprender la importancia de la participación ciudadana en la ciudad del siglo xxi nos hemos fijado en las consecuencias que provoca la ausencia de esa participación, por ejemplo, en un barrio singular de la ciudad de Las Palmas de Gran Canaria. A esta ausencia la denominamos pobreza participativa; una pobreza que, como la pobreza alimentaria o educativa, puede estar relacionada con aspectos cuantitativos (participamos poco) o cualitativos (participamos mal). En un momento en el que las ciudades están sometidas a un proceso acelerado de cambios, la aspiración de la participación en la arquitectura y el urbanismo no debe ser la creación de alternativas a dicha aceleración, sino más bien la creación de «zonas temporalmente autónomas» en las que exista una mayor y mejor participación.

Palabras Clave: participación ciudadana, mediación urbana, indicadores, cooperación, arquitectura social.

ABSTRACT: To understand the importance of citizen participation in the twenty-first century city we analyse the consequences when such participation is absent in the experience of a neighbourhood in Las Palmas, Gran Canaria. We call this absence participatory poverty: it is a poverty that, like food or education poverty, can be quantitative (little) or qualitative (poor participation). At a time when cities are changing rapidly, participation in architecture and urban planning should not aspire to create alternatives to this accelerated 
change, but rather to create "temporarily autonomous zones" where participation is increased and enhanced.

KEYWORDS: citizen participation, urban mediation, poverty indicators, cooperation, social architecture.

RESUM: Per comprendre la importància de la participació ciutadana a la ciutat del segle xxi ens hem fixat en les conseqüències que provoca l'absència d'aquesta participació, per exemple, en un barri singular de la ciutat de Las Palmas de Gran Canaria. A aquesta absència l'anomenem pobresa participativa; una pobresa que, com la pobresa alimentària o educativa, pot estar relacionada amb aspectes quantitatius (participem poc) o qualitatius (participem malament). En un moment en què les ciutats estan sotmeses a un procés accelerat de canvis, l'aspiració de la participació en l'arquitectura i l'urbanisme no ha de ser la creació d'alternatives a aquesta acceleració, sinó més aviat la creació de «zones temporalment autònomes» en què hi haja una major i millor participació.

Paraules Clau: participació ciutadana, mediació urbana, indicadors, cooperació, arquitectura social.

\title{
I. Tiempos acelerados. De lo preventivo a lo paliativo
}

\begin{abstract}
A lgunos autores se refieren a este momento como el de la gran aceleración o era antropocénica (Steffen et al., 2015). Como si se tratara de la persecución de un pelotón ciclista, propuestas como el foro social mundial, el decrecimiento, las ciudades en transición, el procomún, etc., se han ido relevando en estas primeras décadas del siglo XXI en un intento, también acelerado, por aportar experiencias o ritmos alternativos a las corrientes predominantes. Juhani Pallasmaa, citando a David Harvey, Fredric Jameson o Paul Virilio, nos habla de la desaparición del tiempo experiencial en la arquitectura del siglo xx: «Mientras que los edificios y los lugares construidos antes de la modernidad eran documentos de un tiempo benevolentemente lento, la arqui-
\end{abstract}


tectura parece haberse vuelto más rápida, apresurada e impaciente a lo largo de la era moderna» (Pallasmaa, 2015, p. 116).

Si como afirma Bauman, ya hemos pasado de una cultura de aprendizaje y acumulación, a una cultura de la retirada, la discontinuidad y el olvido, en la que cementerios y museos compartirían una misma categoría (Bauman, 2013, p. 151), tal vez va siendo hora de plantearnos el paso de los tratamientos preventivos a los tratamientos paliativos en los que, siendo conscientes del deterioro irreversible (bien sea progresivo o inminente), concentremos los esfuerzos en cuidar lo mejor posible al enfermo, esto es, como aquella estrategia propuesta por Italo Calvino en Las ciudades invisibles, «hacerlo durar y darle espacio». En Los siete mensajeros, breve relato del también escritor italiano Dino Buzzati, la búsqueda metódica e infructuosa de los límites del reino, lleva al protagonista a superar una frontera a partir de la cual pasado y futuro cambian su papel. En cierto momento del relato, deja de tener sentido enviar mensajeros hacia el pasado — para contar sus avances o recibir noticias de un origen ya remoto-. Por el contrario, la inminencia del fin le lleva a enviar sus mensajeros hacia el futuro, consciente de la huida hacia adelante que eso representa, en una metáfora muy similar al clásico quemar las naves atribuido a Alejandro Magno. En ese futuro que todavía no existe es donde podemos plantear lo que Hakim Bey denomina zonas temporalmente autónomas, esto es, experiencias de resistencia a pequeña escala, una «táctica perfecta para una época en la que el estado es omnipotente y omnipresente, pero también lleno de fisuras y grietas» (Bey, 2007, p. 15). Las herramientas que maneja la arquitectura se convierten en imprescindibles para esa huida hacia adelante, que no propone ya soluciones permanentes sino propuestas silenciosas que acompañen a una ciudadanía insurgente (Holston, 1995). Como el propio Bey plantea: «La Revolución está clausurada, pero la insurgencia abierta. En nuestro tiempo no cabe sino concentrar nuestra fuerza en "poderes insurgentes", eludiendo todo entrampamiento en cualquier "solución permanente"». (Bey, 1991). 


\section{Las dimensiones de la participación}

El origen etimológico de la palabra participación —del latín pars-partis: porción o parte y capere: tomar o coger- nos dice que podemos considerar, por un lado, una participación pasiva vinculada a la pertenencia, la vinculación o el arraigo, y una participación activa, vinculada a la creación, a la libertad o a la singularidad. También, en tanto necesidad humana (Max-Neef), como la subsistencia o la protección, podemos encontrar en la participación aspectos cuantitativos y cualitativos. Podemos hablar de mucha o poca participación, esto es, de que participamos más o menos, y también podemos hablar de la calidad de esa participación, esto es, de si es mejor o peor.

Max-Neef considera que la pobreza no se encuentra exclusivamente vinculada con aspectos económicos, sino especialmente con la existencia de necesidades no satisfechas. Existiría una «pobreza de subsistencia (debido a alimentación y abrigo insuficientes); de protección (debido a sistemas de salud ineficientes, a la violencia, a la carrera armamentista, etc.); de afecto (debido al autoritarismo, la opresión, las relaciones de explotación con el medio ambiente natural); de entendimiento (debido a la deficiente calidad de la educación); de participación (debido a la marginación y discriminación de mujeres, niños y niñas y minorías); de identidad (debido a la imposición de valores extraños a culturas locales y regionales, emigración forzada, exilio político, etc.)» (Max-Neef, 1994, p. 43). Igualmente la filósofa y escritora india Vandana Shiva afirma que «[...] la pobreza económica es sólo una de las formas de la pobreza. La pobreza cultural, la pobreza social, la pobreza ética, la pobreza ecológica, la pobreza espiritual son otras formas de pobreza con mayor prevalencia en el así denominado rico Norte, que en el Sur, denominado pobre».

Por lo tanto, la pobreza de la participación tendría que ver tanto con la cantidad, por ejemplo, la falta de participación, que podemos ver de forma más clara en regímenes totalitarios o no democráticos, como con la calidad, esto es, la ausencia de una buena calidad de la participación. En la imagen (fig. 1) que reproducimos a continuación sucede que, cuantitativamente, el $100 \%$ de los presentes en esta concentración de obreros de los astilleros de Blohm und Voss, en Hamburgo en 1936, están participando en la concentración. Sin embargo, después de más de ocho décadas, sabemos que, cualitati- 
vamente, sólo uno de los presentes llevó a cabo una participación de calidad, acorde con la libertad y los derechos humanos. El resto de participantes, cada uno con su propia motivación, estaban ejerciendo una pésima participación, esto es, una mala calidad de la participación.

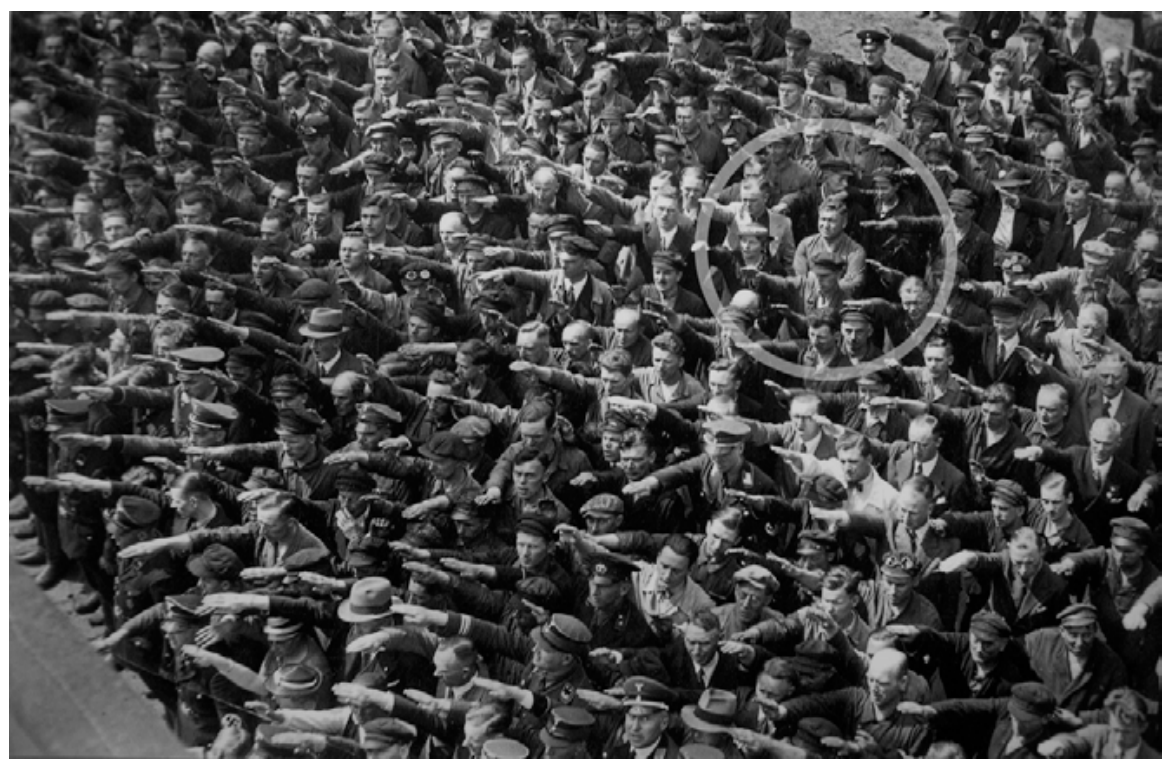

Figura 1. August Landmesser, obrero de los astilleros de Blohm und Voss (Hamburgo, 1936). La fotografía se encuentra expuesta en el centro de documentación Topografía del Terror en Berlín.

En 1969 Sherry R. Arnstein publicó los denominados peldaños o niveles de participación ciudadana. con una escalera que iba de la ausencia absoluta de participación - lo que Arnstein denominaba manipulación o no-participación-, a un estadio máximo de participación denominado control social (Arnstein, 1969). Como bien aclara Eugene Mullan, «[...] no necesariamente se produce una mejora en la participación conforme se va ascendiendo por la escalera. Cada nivel diferente puede ser apropiado según la situación concreta. Lo importante es que la gente tenga la oportunidad de participar en el nivel que satisfaga sus necesidades y que les haga sentir que tienen un control suficiente sobre su entorno» (Mullan, 2005).

La dimensión ciudadana de la participación tiene claros antecedentes en la década de 1960 y más concretamente, en su relación con la consolidación de la ciudad como hábitat indiscutible del ser humano. El fenómeno de la urbanización que toma fuerza en los países de América Latina coincide con una nueva etapa en la historia de la arquitectura en la que, desde diferentes enfoques o 
miradas, se plantea una revisión crítica de los postulados de la arquitectura moderna. Más allá de la arquitectura y el urbanismo, la ciudad comienza a ser estudiada desde una óptica social por autores como Jane Jacobs (1961), Francoise Choay (1965), Henry Lefebvre (1968) o Manuel Castells (1973); desde la antropología por Lévi-Strauss (1962) o Marvin Harris (1962); desde la semiología por Roland Barthes (1961) o Umberto Eco (1962). En esta década la participación recibe también un impulso por parte de muchos pensadores desde diferentes disciplinas como Guy Debord (1958), Ezequiel Ander Egg (1962), Raymond Queneau (1966), Edgar Morin (1969) o Murray Bookchin (1974)

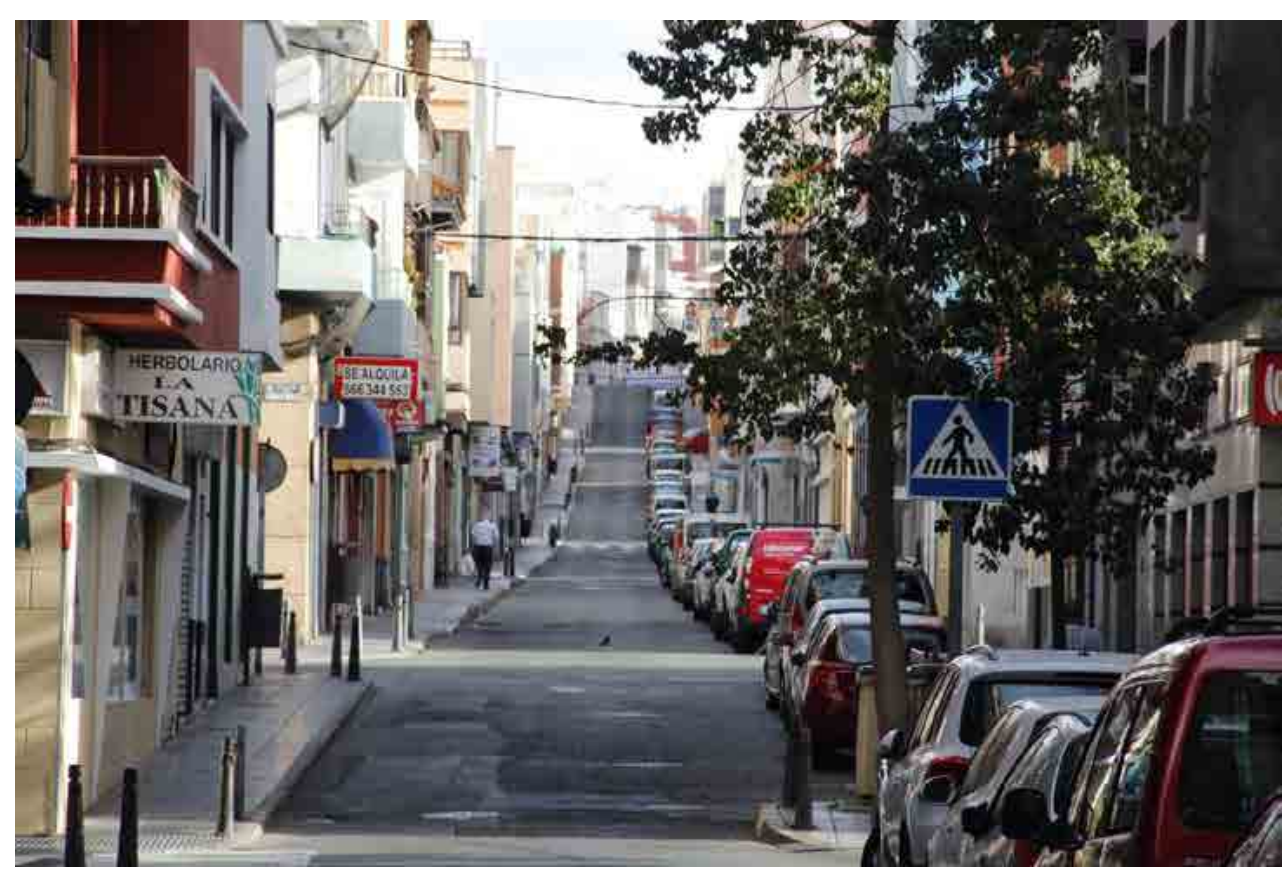

Figura 2. Calle Luján Pérez en el barrio de La Isleta, mayo de 2017.

\section{El barrio de La Isleta como campo de experimentación}

Hasta los años noventa dominaba una concepción normativa en la ordenación de la ciudad y el territorio, heredera directa del urbanismo moderno. Los planes urbanísticos - generales, parciales, especiales- eran los instrumentos básicos para tal fin. En la última década del siglo Xx se añadió una concepción estratégica del urbanismo que aportaba una visión más compleja y multidisciplinar, principalmente para las ciudades. Los planes estratégicos se enfrentaban a la necesidad de situar a las ciudades en el mapa global, fomen- 
tando su competitividad — turística, deportiva, comercial一 o priorizando los aspectos económicos, como la idea de dotarlas de una marca. Es claro que en esta segunda década del siglo XXI ha llegado el momento de una concepción participativa del urbanismo, en la que se incorpore a la ciudadanía en todas las fases, desde la definición de los problemas hasta la toma de decisiones. También sabemos que dicha participación requiere la aplicación de nuevas herramientas inéditas hasta ahora.

Entonces debemos enfrentar por un lado la idea de un mundo que en lo global combina los mejores datos en cuanto a bienestar, esperanza de vida, educación, renta, etc. de toda su historia, con esa gran aceleración que nos acerca al colapso y que nos conmina a una estrategia de retirada decreciente. En lo local debemos abordar la intervención desde cada una de nuestras disciplinas tratando de dar respuestas transdisciplinares a los problemas concretos que acucian a nuestras ciudades. Para comprender el trabajo que estamos desarrollando en el barrio de La Isleta, es importante realizar un acercamiento tanto desde la arquitectura como desde otras disciplinas, como la antropología urbana o la sociología.

Uno de los primeros aspectos que debemos abordar es el redescubrimiento de las comunidades y las redes de proximidad. A una lógica más antropológica vinculada tal vez con los lazos de parentesco, de amistad y de vecinazgo, desde la arquitectura y el urbanismo podemos incorporar la dimensión espacial a esa ecuación. De esta forma, al concepto de comunidad, definida como «grupo o red informal con vínculos entrelazados de conocimientos personales de larga duración que proporcionan apoyo, información, sentido de pertenencia e identidad social» (Costa, 1999, p. 149), le incorporamos una dimensión urbanístico-espacial, que a su vez tiene que ver con el tejido urbano consolidado, con la escala física y con el estadio evolutivo en el que se encuentra. La incidencia sobre los habitantes del barrio de La Isleta en las últimas décadas de aquello que Sennett define como «comunidad de la abundancia» en la que «la necesidad de interacción social, la necesidad de compartir, deja de ser una fuerza motriz...»o en la que «los individuos pueden concebir fácilmente su vinculación social en función de su semejanza mejor que de su mutua necesidad», puede transmitirnos la idea de barrio sin comunidad (Sennett, 1975, p. 68). 
Frente a un enfoque más tradicional del concepto de comunidad, estrechamente vinculado al barrio, el enfoque que queremos trasladar en La Isleta se acerca más a lo que Costa (1999) denomina sociedad de barrio. En este punto queremos aclarar que nuestra propuesta se aleja de los análisis cualitativos, para volver a incidir, al menos de forma parcial, en valores cuantitativos, que nos permitirían, gracias a los medios técnicos con los que contamos actualmente, abordar nuevas lecturas transdisciplinares y también compartidas con la propia sociedad.

\section{Tres ejemplos}

Indudablemente, la ciudad, como hábitat del ser humano, también representa un lugar de encuentro de múltiples posturas enfrentadas. Ya sean diferencias políticas, religiosas, de lenguaje, de raza, de conducta, etc., la ciudad tiene inevitablemente ante sí la ingente tarea de unificar posturas y aplacar conflictos. El triángulo — de origen greco-romano- que enmarca el término ciudad: polis, urbs y civitas (Alomar, 1961; Delgado, 1999, p. 192; Capel, 2003) o en otra formulación: príncipe, arquitecto, pueblo (De Manuel, 2005) nos sirve para simplificar los actores que intervienen en ella. A continuación utilizaremos esa clasificación para presentar tres ejemplos de menor entidad, que dan muestra de la forma de intervenir de la lógica tecno-instrumental (Raposo, 2009) y de la necesidad de la participación ciudadana para intervenir en esa «sociedad de barrio», caracterizada por su complejidad (Morin, 2004).

El primer ejemplo se refiere a la intervención directa de la administración pública, vinculada estrechamente con la lógica política: ¿por qué entre los años 2012 y 2015 se han seguido reponiendo aceras que no alcanzan los 50 centímetros de ancho? El segundo afecta a la lógica técnica: ¿por qué se siguen pintando los pasos de cebra a 5 metros del lugar por el que cruzan los vecinos? Y el tercero a la lógica ciudadana: ¿por qué se continúa aparcando en las aceras? Se trata de responder a estas preguntas como medio para mostrar que cualquier solución implica la necesidad de una acción compartida de todos los actores, además de una verdadera voluntad transformadora principalmente de aquellos llamados a tomar las decisiones. 
Reposición de aceras

A la pregunta de por qué en la segunda década del siglo XXI se siguen construyendo aceras que tienen $30 \mathrm{~cm}$ de ancho, la respuesta es que las propias instituciones públicas, en algunos casos, no aplican las normas en vigor, sino que se ven abocadas a actuar siguiendo la lógica de normas no escritas que son aceptadas por todos los actores implicados. En el caso de la reposición de aceras en el barrio, la ausencia de una política estratégica o de una planificación urbanística más compleja, que incorpore por ejemplo mecanismos de participación ciudadana, ha llevado a los diferentes equipos de gobierno que se han sucedido en la alcaldía del municipio, y los correspondientes equipos técnicos, a replicar algunos de los errores del pasado sin margen para reconducirlos. El caso de la reposición de aceras nos permite abordar algunas derivas de este fenómeno.

Después de casi 80 o 90 años sin intervenir integralmente en la mejora de las vías del barrio, a partir de 2010 se comenzó la mejora de las redes de saneamiento, de abastecimiento de aguas, el asfaltado de las calles y la reposición de aceras. Precisamente nos interesa analizar con más detenimiento la reposición de aceras porque en nuestra opinión visibiliza de una forma clara las dificultades a las que se enfrenta la acción pública cuando no se incorporan mecanismos de participación ciudadana en la toma de decisiones. Para resumir la lógica subyacente diremos que en La Isleta existen numerosos casos de aceras que incumplen la normativa de accesibilidad en cuanto a la dimensión (fig. 3 y 4).

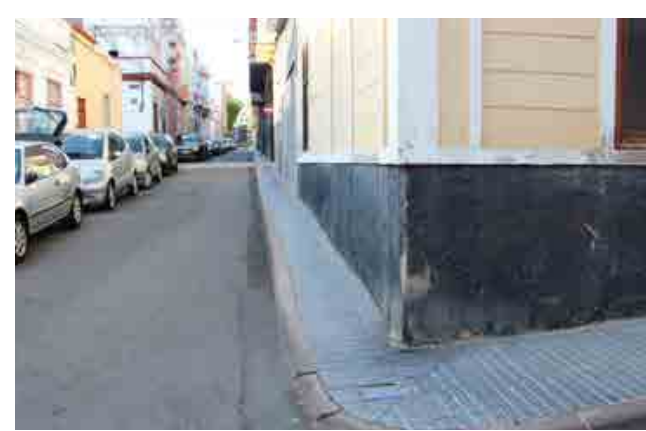

Figura 3. Acera de la calle Tanausú, esquina calle Tauro. Acera repuesta el año 2010, barrio de La Isleta.

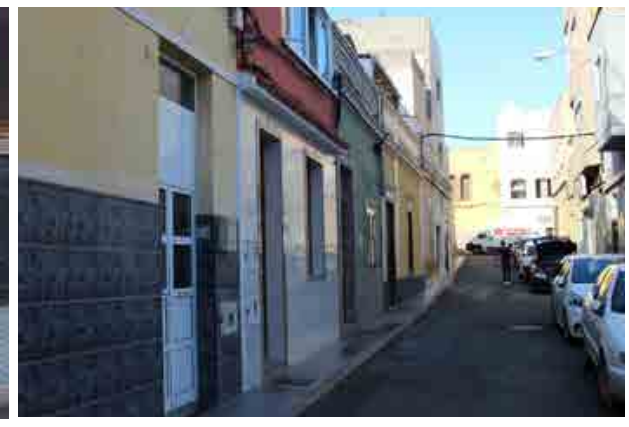

Figura 4. Acera calle Romeral, barrio de La Isleta. 
Es claro que el cumplimiento estricto de la normativa general y municipal correspondiente al diseño de los espacios públicos implicaría una modificación de la sección de la vía y, con toda seguridad, en la mayor parte de los casos, la eliminación de una banda de aparcamientos. En la voluntad de los técnicos de la administración se presupone el deseo y la obligación del cumplimiento de la normativa vigente, al mismo tiempo que el cumplimiento de los plazos de ejecución de la obra. Dado que la supresión de aparcamientos podría conllevar el rechazo de muchos vecinos, llegando incluso a la paralización de las obras, en La Isleta se optó en esta ocasión por no afrontar el problema de la accesibilidad y renovar las aceras replicando las dimensiones en muchos casos insuficiente que éstas ya tenían.

Se trata sin duda de una oportunidad perdida. Pero ¿son los vecinos del barrio contrarios a la eliminación de aparcamientos en superficie para hacer las calles más accesibles? En nuestra opinión, en esta ocasión ni siquiera se ha querido plantear el problema, en aras de terminar las obras en el corto plazo. Ciertamente podemos decir que la opinión mayoritaria de los vecinos se inclina más por la supremacía del coche frente a la mejora de la accesibilidad y la calle. La necesidad de aparcamiento se impone todavía a la necesidad de un uso más racional de la calle. En este caso, desde el Ayuntamiento, tanto su dirección técnica como su dirección política, han atendido a la expresión mayoritaria del vecindario del barrio, incluso por encima de cuestiones legales o de justicia social.

Éste es un primer ejemplo de respuesta condicionada en forma de cadena a la lógica del corto plazo. Cumplir la normativa de accesibilidad obligaría a ensanchar las aceras y por lo tanto a eliminar aparcamientos en superficie. Como esa medida podría generar protestas que pondrían en peligro la ejecución de las obras en el tiempo previsto, el propio Ayuntamiento decide saltarse la norma. Para no volver a caer en la excepcionalidad, cuando no ilegalidad, y revertir una situación en la que hay minorías que no han sido tenidas en cuenta — niños, ancianos, personas con diversidad funcional— una de las soluciones posibles viene de la mano de la participación ciudadana y consiste, desde este momento, en abrir debates y educar a la ciudadanía para que en el medio plazo, después de escuchar a esas otras miradas, se produzca un 
cambio que potencie una mirada más inclusiva hacia la accesibilidad, frente a la mirada excluyente del coche privado ocupando la vía pública.
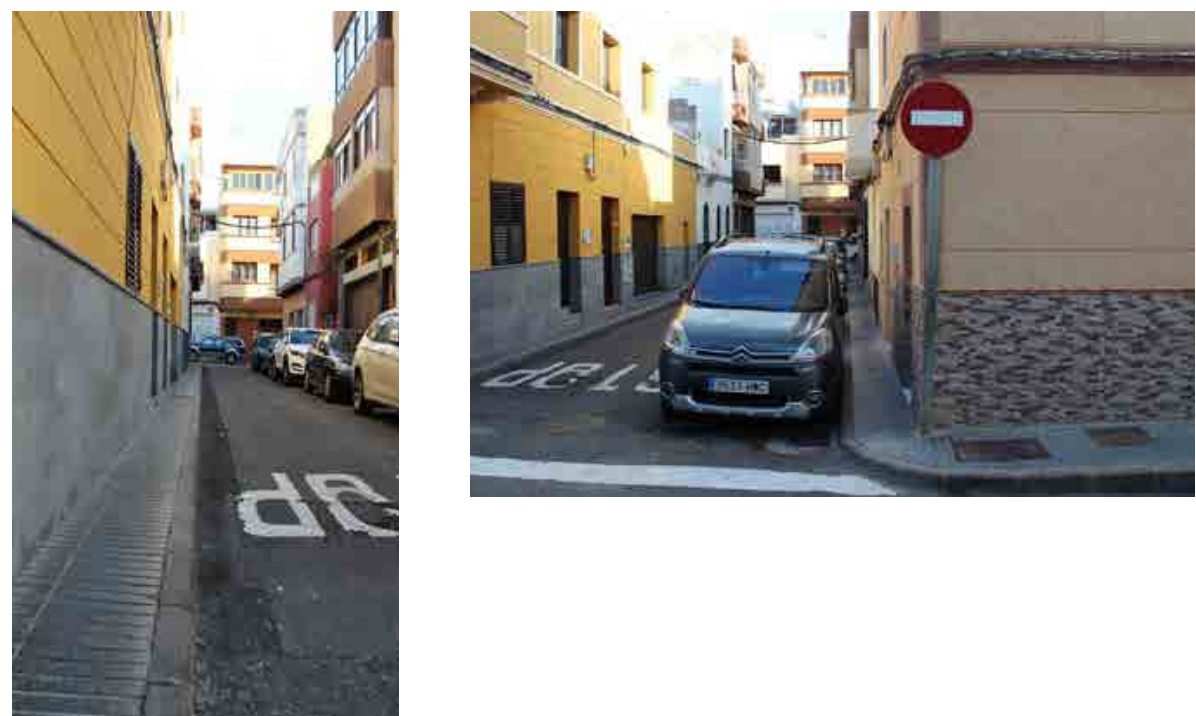

Figura 5. Acera calle Juan Sebastián Elcano, barrio de La Isleta.

Pasos de cebra

¿Por qué en algunos cruces de calles el $100 \%$ de los vecinos no cruza por el paso de cebra? En lo que se refiere a los pasos de cebra en el interior del barrio, la lógica técnica se impone sobre la lógica política — que apenas interviene en este tema- y el sentido común de la ciudadanía — que directamente incumple la norma. Aunque se trate de un tema aparentemente menor, la aplicación de la normativa sobre la ubicación de los pasos de cebra en muchos barrios de la ciudad de Las Palmas es una verdadera invitación a saltarse la norma para los que diariamente transitan por sus calles.

Al contrario de lo que sucedía con las aceras, que era el propio Ayuntamiento el incumplidor, en este caso el técnico municipal encargado de ubicar los pasos de cebra ha decidido aplicar la normativa en vigor, independientemente de cualquier consideración sobre el lugar, sobre la sección de las calles, o los hábitos de los vecinos. Cuando el $100 \%$ de esos habitantes no cruzan habitualmente por dichos pasos de cebra — situados a 5 metros del cruce obligando a un desplazamiento innecesario en este tipo de calles- podemos afirmar que correspondería a los técnicos municipales poner en crisis la normativa existente (fig. 6 y 7). Tantos vecinos no pueden estar equivocados y 
decidir saltarse las normas sistemáticamente. En este caso tenemos un ejemplo claro de que la participación ciudadana podría hacer avanzar el marco normativo en vigor.

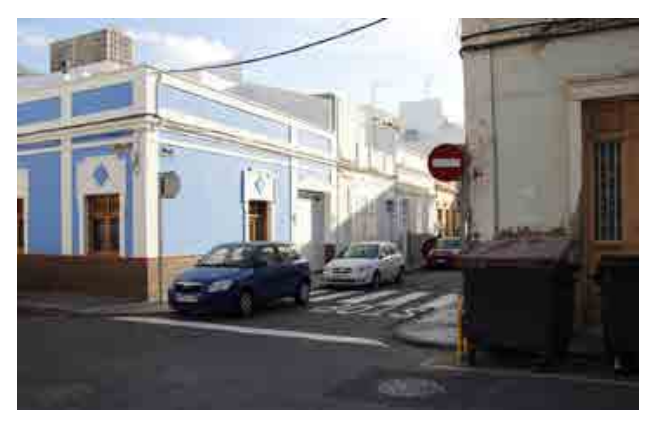

Figura 6. Paso de peatones en la calle Fontanales, barrio de La Isleta.

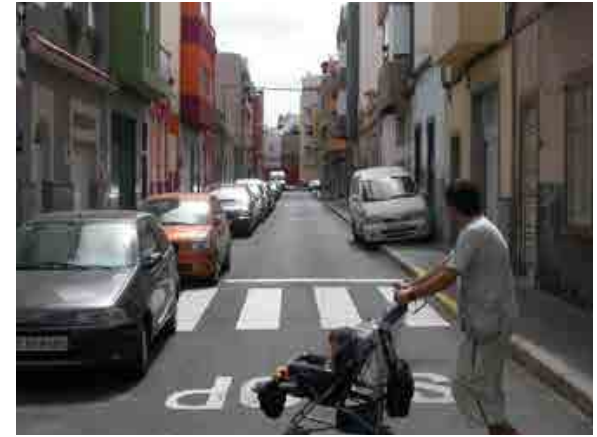

Figura 7. Paso de peatones en la calle Tauro, barrio de La Isleta.

Aparcar en las aceras

¿Por qué se aparca en las aceras de algunas calles del barrio? La primera respuesta que daría cualquier vecino del barrio sería inmediata: porque no hay aparcamientos. Obviamente, si las calles del barrio contaran con espacio ilimitado de aparcamiento adecuado y gratuito, éstos serían sin duda ocupados antes que las aceras. Vamos a intentar analizar este fenómeno. En primer lugar debemos decir que dentro del carácter ilegal de aparcar en la acera, existen una serie de normas no escritas que se respetan escrupulosamente entre los vecinos y vecinas del barrio. La primera y más clara consiste en no interrumpir el paso de los coches por la calzada, lo que lleva a que en algunas calles no se aparque porque no hay espacio suficiente para ello. La segunda norma consiste en no interrumpir la salida de las casas, tanto de las puertas principales como de los garajes con vado horario o permanente. El incumplimiento de cualquiera de estas normas acabaría con este tipo de aparcamientos ya que los afectados inmediatamente llamarían a la policía. De hecho, la proliferación de garajes con vado permanente ha reducido considerablemente la superficie ocupada por estos pseudo-aparcamientos (fig. 8 y 9). 

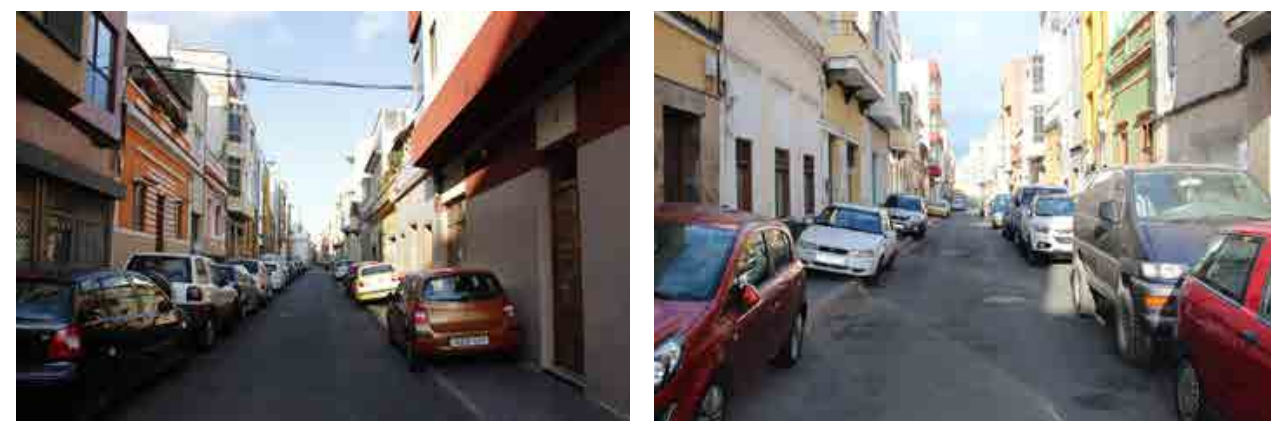

Figuras 8 y 9. Coches aparcados en la acera de la calle Tauro, barrio de La Isleta.

Cuando, por alguna de las circunstancias señaladas, finalmente debe acudir la policía, el espectáculo es dantesco porque se daría la tesitura de que los agentes deben proceder a denunciar no sólo al vehículo que ha originado la llamada, sino a todos los que están en la misma situación a lo largo de toda la calle. Se da la circunstancia de que la propia policía prefiere llegar a solucionar el problema por una vía amistosa antes de proceder a denunciar este problema ya enquistado en algunas calles del barrio. Tanto los vecinos, como la policía coinciden en evitar medidas punitivas, como las multas, o coercitivas, como la instalación de bolardos, para dar una solución definitiva al problema.

¿Cómo hacer cumplir la ley? Volvemos en este caso a encontrarnos con el duelo entre la necesidad y la cultura. Mientras la necesidad de aparcamientos tenga mayor aceptación social que la lógica cultural del uso peatonal de las aceras — por niños, personas mayores o con movilidad reducida— será muy complicado erradicar este problema. La participación ciudadana vuelve a ser una de las vías para que cobren fuerza y presencia las voces que reclamen ese uso peatonal de las aceras y al mismo tiempo censuren la ilegalidad de los coches en las aceras. Es sin duda una tarea de concienciación que precisa tiempos más largos.

\section{Mediación urbana. La incorporación de la participación ciudadana}

Siguiendo algunos de los nuevos principios del urbanismo propuestos por François Ascher (2004), como ese urbanismo de resultados, más crea- 
tivo y proactivo que el actual, existen aspectos que el urbanismo heredado o heredero del siglo xx aún no ha sabido incorporar. Hablamos, como enumera Skolimowski, por ejemplo, de la cooperación y la creatividad, o bien de la solidaridad: «La filosofía participativa exige el redescubrimiento de la participación como un modo de estar en el mundo [...] implica un redescubrimiento del coraje, del valor de ser y devenir [...] un redescubrimiento de la propia mente» (Skolimowski, 2016, p. 450). Con respecto a la solidaridad, también en el barrio de La Isleta estamos desarrollando un proyecto de investigación que tiene como objetivo fundamental contribuir, a través de la cartografía, a devolver a la población la información y el conocimiento del que disponen tanto las administraciones públicas, como los técnicos o las empresas de servicios. Se trata de propiciar también una comunicación más fluida entre todos estos actores. A través de lo que denominamos Indicadores Urbanos Activos (Díaz-García, 2011; 2016) se pretende fomentar la cooperación institucional y la creación de redes locales como base de desarrollo territorial. Esto implica facilitar el intercambio y el uso activo de dicha información por parte de los profesionales de las propias administraciones públicas, en un verdadero acto de cooperación interadministrativa. El cambio hacia una arquitectura y un urbanismo participativo implica volver a plantearse la intervención pública incorporando esta dimensión desde el principio. Esto también implica la aparición de nuevas herramientas y nuevos principios, así como nuevos perfiles profesionales.

Salvo casos como el del arquitecto argentino Rodolfo Livingston (2004), los arquitectos y arquitectas todavía no han incorporado la participación ciudadana en su quehacer profesional. Sigue existiendo un gran salto entre el lenguaje de la arquitectura y lo que podemos denominar el sentido común. Aunque en parte el marco legal y normativo, o el propio lenguaje de la arquitectura, podrían justificar este alejamiento, no hay, sin embargo, razones para que en ese enorme vacío no existan profesionales encargados de mediar o traducir entre ambos lenguajes. La mediación urbana se presenta como un nuevo campo de acción de los arquitectos.

Recientemente, en el año 2016, el Ayuntamiento de Madrid ha puesto en marcha una formación especializada de mediadores urbanos comunitarios, en el que formará a «personas con capacidad para la dinamización comunita- 
ria, la activación de la cultura y con capacidad creativa y transformadora de su territorio». Estos profesionales, en primer lugar, tendrán que conocer el medio en el que se mueven y ser capaces de elaborar un mapa de actores en cada situación a la que se enfrenten. El segundo cometido se refiere a conocer el uso de las herramientas metodológicas o las técnicas concretas de participación ciudadana. La tercera capacidad inherente a esta especialización consiste en estructurar la información obtenida tanto con los mapas como con las técnicas. Por último, y en este caso se ajustaría adecuadamente a la formación del arquitecto, debe tener la capacidad para elaborar diferentes escenarios o alternativas que puedan nutrir el debate con carácter prospectivo, esto es, anticipando posibles caminos por los que avanzar en la resolución de los conflictos. Este mediador urbano, o city manager, debe ser capaz de intervenir o mediar en los conflictos con la habilidad de entender, clarificar, ordenar y proponer, en un tiempo limitado, alternativas posibles y creíbles que serían el resultado del propio proceso participativo.

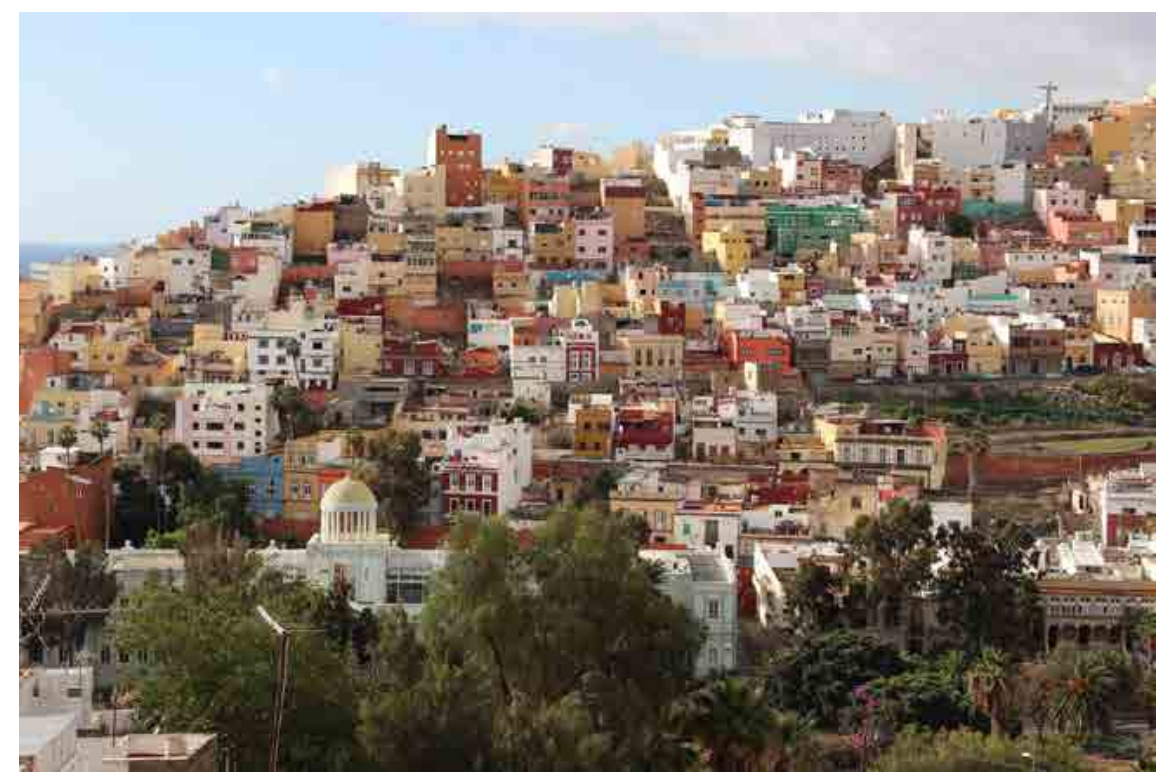

Figura 10. Ejemplo de ciudad informal, Risco de San Juan, Las Palmas de Gran Canaria.

En un momento en el que las ciudades están sometidas a procesos acelerados de cambios, la aspiración de la participación en la arquitectura y el urbanismo no debe ser la creación de alternativas a dicha aceleración, ni siquiera 
su ralentización, sino más bien la creación de «zonas temporalmente autónomas» en las que exista una mayor y mejor participación, y al ritmo que dicha participación demande.

\section{Referencias}

Alomar, G. (1961). Sociología urbanística. Madrid: Aguilar.

Arnstein, S. R. (1969). «A Ladder of Citizen Participation», JAIP, Vol. 35, No. 4, July, pp. 216-224.

Ascher, F. (2004). Los nuevos principios del urbanismo. Madrid: Alianza.

Bauman, Z. (2013). Vidas desperdiciadas. La modernidad y sus parias. Barcelona: Paidós.

BEY, H. (1991). La zona temporalmente autónoma.

Blundell, P., Petrescu, D. y Till, J. (2005). Architecture and participation. Londres: Taylor \& Francis.

CAPEL, H. (2003). «Los problemas de las ciudades. Urbs, civitas y polis». En Capel, Horacio (coord.): Ciudades, arquitectura y espacio urbano, Fundación Cajamar (Serie «Mediterráneo Económico», nº 3) Almería, pp. 9-24.

Da Costa, A. F. (1999). Sociedade de bairro: dinâmicas sociais da identidade cultural. Oeiras: Celta Editora

Delgado, M. (1999). El animal público. Anagrama: Barcelona.

De Manuel, E. (2005). «La construcción del triángulo de la cooperación. El pueblo, el arquitecto y el príncipe», conferencia pronunciada en Las Palmas de Gran Canaria, 2005.

- (2010). «Construyendo triángulos para la gestión social del hábitat». Hábitat y sociedad, 1, noviembre, pp. 13-37.

Díaz-García, V. (2011). «Indicadores Urbanos Activos (IURA). Un camino hacia el Índice de Desarrollo Urbano». Proceedings of $7 \mathrm{VCT}$, Lisbon, Portugal, 11-13 October 2011, p. 529. Recuperado de https:// upcommons.upc.edu/bitstream/handle/2099/15891/92_Indicadores Urbanos Activos (IURA).pdf

— Cárdenes Macías, D., Delisau Suárez, S., Febles Arévalo, T., Santana Santana, J., V Alido Medina, M. Y. (2016). «Iura-la Isleta: Hacia una cartografía social». Congreso ArCaDiaIV. Recuperado de https:// drive.google.com/file/d/0B3aUsRnv0r_jTnNoRkc4Y1dJNm8/ view, p.242-252 
Giddens, A. (1999). Consecuencias de la modernidad. Madrid: Alianza editorial.

Hall, E. T. (2003). La dimensión oculta. México: Siglo XXI.

Holston, J. (1995). «Spaces of Insurgent Citizenship». Planning Theory, 13, pp. 37-56.

KAPUSCINSKI, R. (2007). Encuentro con el otro. Barcelona: Anagrama.

Livingston, R. (2004). Arquitectos de la comunidad. El método. Buenos aires: Kliczkowski.

Martín Hernández, M. (1997). La invención de la arquitectura. Madrid: Celeste.

- Díaz, V. y Rodríguez, E. (2014). Un modo de afrontar la ciudad africana. Madrid: La Catarata / Casa África.

Martínez López, M. (2005). «Urbanismo, participación ciudadana y planificación estratégica de ciudades». Recuperado de http://www.miguelangelmartinez.net/IMG/pdf/2005_Plan_estrategica_ciudades.pdf

Max-Neef, M. (1994). Desarrollo a escala humana. Conceptos, aplicaciones y algunas reflexiones. Barcelona: Icaria.

Moix, Ll. (2010). Arquitectura milagrosa. Hazañas de los arquitectos estrella en la España del Guggenheim. Barcelona: Anagrama.

Montaner, J. M., y Muxí, Z. (2011). Arquitectura y política. Ensayos para mundos alternativos. Barcelona: Gustavo Gili.

Morin, E. (2004). Introducción al pensamiento complejo. Barcelona: Gedisa.

Mullan, E. (2005). «De las islas a las tierras altas de Escocia: experiencias de participación ciudadana en la práctica». En Herrero, L. F. (ed.): Participación ciudadana para el urbanismo del Siglo XXI, ICARO, Valencia, p.72.

Pallasmaa, J. (2015). «Habitar el tiempo». Habitar. Barcelona: Gustavo Gili.

Pelli, V. (2006). Habitar, participar, pertenecer. Acceder a la vivienda - incluirse en la sociedad, Buenos Aires: Nobuko.

Raposo Moyano, A. (2009). «La racionalidad tecno-instrumental en el accionar político-administrativo del estado. Elementos para un modelo descriptivo». Santiago de Chile: Revista INVI, noviembre 1998, 13(35).

Romero, G. Y Mesías, R. (coord.) (2004). La participación en el diseño urbano y arquitectónico en la producción social del hábitat, México D. F.: CYTED-HABYTED. 
Santos, B. De S. (2010). Para descolonizar Occidente. Más allá del pensamiento abismal. Buenos Aires: CLACSO.

SAsSen, S. (2010). Una sociología de la globalización. Madrid: Katz editores.

SECCHI, B. (2015). La ciudad de los ricos y la ciudad de los pobres. Madrid: La Catarata.

Sennett, R. (1975). Vida urbana e identidad personal. Barcelona: Península.

- (2009). El artesano. Barcelona: Anagrama.

- (2012). Juntos. Rituales, placeres y política de cooperación. Barcelona: Anagrama.

Sкolimowski, H. (2016). La mente participativa. Girona: Atalantap.

STEFfen, W. $\boldsymbol{E T} \boldsymbol{A L}$. (2015). «The Trajectory of the Anthropocene: The Great Acceleration». En Anthropocene Review. Vol. 2 (1), pp. 81-98.

SudJIC, D. (2007). La arquitectura del poder. Barcelona: Arial.

Turner, J. (1977). Vivienda, todo el poder para los usuarios: hacia la economía en la construcción del entorno. Madrid: Blume.

Verdaguer, C. (2011). «Marco conceptual: la participación ciudadana como instrumento de sostenibilidad urbana». Recuperado de http://www. diba.cat/c/document_library 